\title{
Demonstration of a real-time interferometer as a bunch-length monitor in a high-current electron beam accelerator
}

\author{
J. Thangaraj, ${ }^{1, \text { a) }}$ R. Thurman-Keup, ${ }^{1}$ J. Ruan, ${ }^{1}$ A. S. Johnson, ${ }^{1}$ A. Lumpkin, ${ }^{1} \mathrm{~J}$. \\ Santucci, ${ }^{1}$ T. Maxwell, ${ }^{1}$ G. Andonian, ${ }^{2,3}$ A. Murokh, ${ }^{3}$ M. Ruelas, ${ }^{3}$ and A. Ovodenko ${ }^{3}$ \\ 1) Fermi National Accelerator Laboratory, Batavia, IL 60510, \\ U.S.A \\ ${ }^{2)}$ Dept of Physics and Astronomy, University of California, Los Angeles, CA 90095, \\ U.S.A \\ ${ }^{3)}$ RadiaBeam Technologies, Santa Monica, CA 90404, U. S. A
}

A real-time interferometer (RTI) has been developed to monitor the bunch length of an electron beam in an accelerator. The RTI employs spatial autocorrelation, reflective optics, and a fast response pyro-detector array to obtain a real-time autocorrelation trace of the coherent radiation from an electron beam thus providing the possibility of online bunch-length diagnostics. A complete RTI system has been commissioned at the A0 photoinjector facility to measure sub-mm bunches at $13 \mathrm{MeV}$. Bunch length variation $(\mathrm{FWHM})$ between $0.8 \mathrm{ps}(\sim 0.24 \mathrm{~mm})$ to $1.5 \mathrm{ps}(\sim 0.45 \mathrm{~mm})$ has been measured and compared with a Martin-Puplett interferometer and a streak camera. The comparisons show that RTI is a viable, complementary bunch length diagnostic for sub-mm electron bunches.

a)jtobin@fnal.gov 


\section{INTRODUCTION}

An important figure of merit in modern accelerators and x-ray free-electron lasers is the electron beam bunch length. The generation and preservation of ultrashort, low-emittance electron bunches is required by these facilities ${ }^{1}$. Therefore, it is critical to measure the beam bunch length throughout the accelerator. Ideally, the bunch length should be measured in a single shot without any interruption to the accelerator operation. Although there are many methods to measure the bunch length, such as electro-optical sampling ${ }^{2}, \mathrm{RF}$ deflecting cavities $^{3}$, streak camera, and RF-zero-phasing techniques ${ }^{4}$, these methods are either invasive to beam operations, costly or require multiple shots for averaging. Therefore, a non-invasive, real-time, single-shot diagnostic is needed. The real-time interferometer (RTI) described in this work can in principle address all these needs and could be a viable, complementary bunch-length diagnostic for sub-mm electron bunches ${ }^{5}$.

Interferometers are routinely used in accelerators to measure the autocorrelation of the beam-emitted radiation, which contains the bunch-length and bunch-profile information ${ }^{6}$. A major drawback of using a scanning interferometer is that it takes several minutes to scan one mirror to record an autocorrelation trace and involves multiple shots. The RTI operates in single-shot mode and can record the autocorrelation trace in real-time. We present the first results from commissioning the RTI at the Fermilab A0 photoinjector (A0PI) and provide direct comparison to results from a scanning interferometer as well as a streak camera. The existing infrastructure at the A0PI includes an emittance exchange beamline ${ }^{7}$ that provides tunable, sub-mm bunches and a high-repetition rate $(1 \mathrm{MHz})$ pulse train. This gives the flexibility to vary the bunch length and the number of bunches to test the RTI over

a wide range of beam parameters. The RTI constructs the autocorrelation of the coherent transition radiation (CTR) emitted as the charged-particle beam transits the vacuum-foil interface $^{8}$. The RTI measurements can be made non-invasive by using a different coherent source such as coherent synchrotron radiation, coherent edge radiation, etc. Other singleshot bunch-length measurements based on spatial autocorrelation (electro-optic sampling or pyrocamera) or on spectral measurements using gratings have been reported ${ }^{9-11}$, but the techniques reported here require no laser or gratings. 


\section{CONCEPT OF THE REAL-TIME INTERFEROMETER}

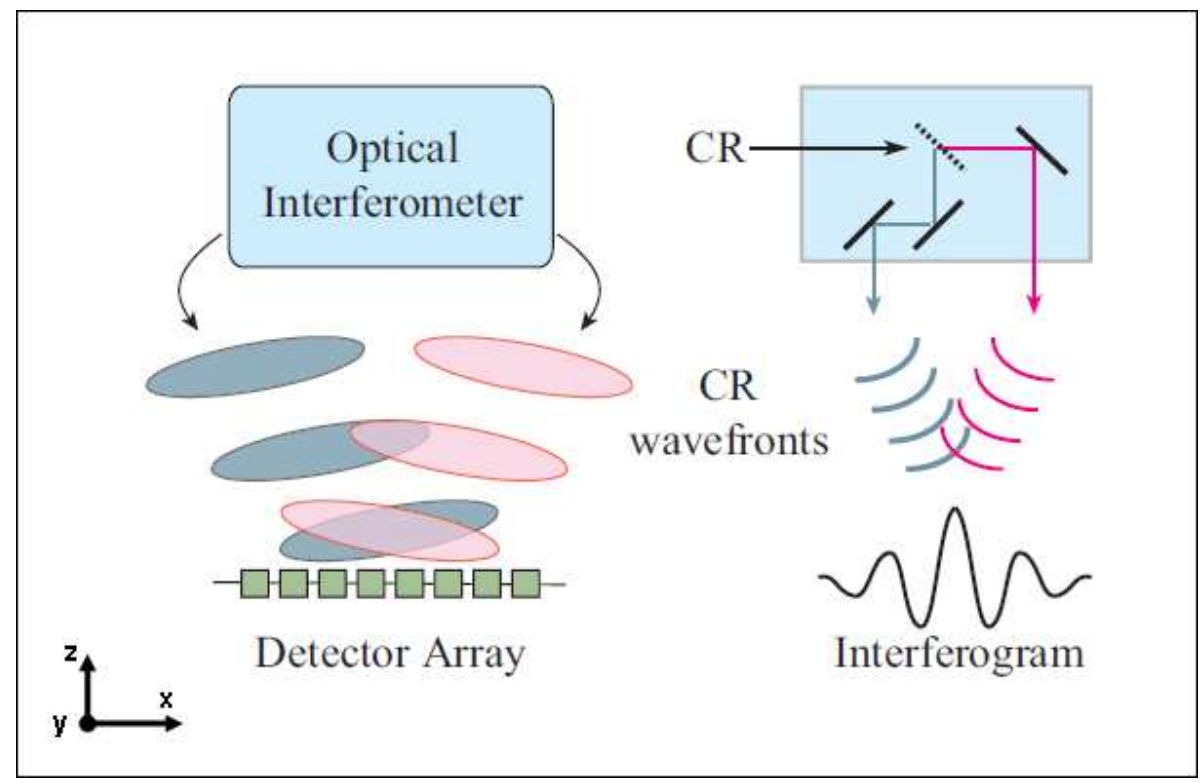

FIG. 1. Operating principle of a spatial auto-correlator. The input beam is split into two signals and they are recombined on a detector plane at an angle. At each point along the detector, the wavefronts of the two beams interfere with each other with a fixed time delay set by the angle. This is equivalent to a classical autocorrelation signal. The left figure shows the spatial autocorrelation of optical laser pulses using a detector array while the schematic on the right shows the spatial autocorrelation using the broadband coherent radiation (CR) signal from an electron beam.

The RTI relies on the spatial autocorrelation of a split signal ( c.f. Fig. 1) where the two beams recombine on the plane of a detector array at a small angle. This differs from the standard interferometer, where the two beams are split and recombined after introducing a time delay (path length difference) to one of the beams. The autocorrelation function for the single-shot (spatial) interferometer is given by:

$S(x) \sim \int d y\left[\int d t \vec{E}(x, y, t) \vec{E}(x, y, t-\theta x)\right]$ where we consider the detector to lie along the $\hat{x}$ axis, one split beam traverses along the $\hat{z}$-axis and the other at a small angle $\theta$ with respect to the $\hat{z}$-axis, and the two beams are focused linearly along the $\hat{z}$-axis. Here $\hat{x}, \hat{y}, \hat{z}$ represent unit vectors along each co-ordinate axis. The real part of the Fourier transform of this autocorrelation function yields the intensity spectrum according to Parseval's theorem ${ }^{12}$. The RTI generates the beam-based coherent radiation autocorrelation function according to this equation. 


\section{A. Diagnostic Layout}

The RTI shown in Fig. 2 employs reflective optics due to the low power levels and frequency range of the emitted radiation. Because CTR is radially polarized, a wire-grid polarizer is used to select the vertical component. The radiation is then sent to the interferometer where it meets a wedge splitter and gets separated into two lobes. One of the lobes travels directly to a cylindrical mirror after reflecting from a few mirrors, while the other lobe is directed to a mirror mounted on a remotely movable stage, which was added at Fermilab for the test. Mounting the mirror on a movable stage makes the path length identical for both the lobes - a significant help in aligning the RTI for different mixing angles. After the two lobes travel through equal path lengths, the pulses are recombined at a small angle at the cylindrical mirror. Finally, the cylindrical mirror focuses the beams in one dimension to create a line focus at the pyrodetector at the set angle. The angle of overlap between the two lobes is controlled by a small mirror mounted on an 'angle-adjustable' mount. The angle of overlap determines the path length difference between the pulses necessary for the interferometry. Further details about the RTI are mentioned elswhere ${ }^{5}$.

\section{B. The Pyrodetector}

The most important development for the RTI was a suitable terahertz detector array. For the bunch lengths of the A0PI electron beam, the emitted CTR is peaked in the sub-THz regime. Pyro-electric detector arrays provide a solution with adequate sensitivity in the $\mathrm{THz}$ regime. The RTI detector array contains 32 elements. Each element is $500 \mu \mathrm{m}$ by $1 \mathrm{~mm}$ and consists of a monolithic, $25 \mu \mathrm{m}$ thick $\mathrm{LiTaO}_{3}$ crystal coated with chromium to increase the sensitivity in the 0.2-3.0 $\mathrm{THz}$ range. The detector channels are set up with a $\mathrm{RC}$ time constant of $\sim 100 \mu \mathrm{s}$; the rise time is less than $10 \mu \mathrm{s}$. The lowest range that could be set at full scale was $2 \mathrm{~nJ}$, with a maximum of $2 \mu \mathrm{J}$. The analog output of all 32 channels requires $5 \mathrm{msec}$ (or $150 \mu \mathrm{s}$ per channel).

\section{EXPERIMENTAL SETUP}

In this section we describe the A0 Photoinjector (A0PI) facility where the RTI was tested. As shown in the schematic in Fig. 3, the A0PI facility consists of a 1.5-cell normal conducting 
L-band RF photocathode gun driven by the frequency quadrupled, UV component of a Nd: glass drive laser. The L-band RF gun is followed by a superconducting booster cavity, which accelerates the e-beam to $14 \mathrm{MeV}$. After acceleration, the beam is sent through the doglegs (D1 D2 D3 D4) followed by a quadrupole triplet and a spectrometer (XS4). Between the doglegs is the deflecting cavity, which is a liquid-Nitrogen-cooled five-cell copper cavity operating on the $T M_{110}$ mode at $3.9 \mathrm{GHz}$. This beamline is called the emittance exchange line.

One of the experimental goals of the A0PI facility is to test and develop the concept of emittance exchange (EEX) in which the transverse phase space of the electron beam is exchanged with its longitudinal phase space ${ }^{7}$. To observe the emittance exchange, the bunch length, among other beam parameters, has to be measured before and after the EEX process. An important advantage of the EEX for our experiment is the ability to change the bunch length of the electron beam at X24 just by varying the upstream quadrupoles. A He-Ne laser beam was injected upstream of X24 to align the path to and through the RTI. This was a crucial step in setting up the RTI. Currently a Hamamatsu C5680 streak camera, Martin-Puplett interferometer, and a helium-cooled bolometer are available to measure the bunch length of the electron beam at X24 ${ }^{13}$. Typically, the bunch length is measured via the autocorrelation of the CTR transmitted through a single-crystal diamond window as the beam hits an aluminum foil. The CTR radiation is then sent through a wire-grid polarizer (15 $\mu \mathrm{m}$ diameter gold-plated tungsten wires spaced by $45 \mu \mathrm{m}$ ), which selects the vertical component of the CTR that is transported either to the Martin-Puplett interferometer or the RTI.

In this work, we focus on the bunch length measurements done at location X24, which is about $60 \mathrm{~cm}$ after the EEX beamline, using the RTI. The RTI interferogram signal was recorded in a Lecroy WavePro 740Zi-A scope to obtain the autocorrelation in real-time after background processing.

\section{EXPERIMENTAL RESULTS}

The RTI was tested and compared to two other bench-marked diagnostics at the A0PI. After obtaining an autocorrelation trace using CTR from X24, the RTI measurements were subsequently compared with those from a Martin-Puplett interferometer and a streak cam- 
era. This section describes the methods and results.

\section{A. Autocorrelations}

A typical autocorrelation trace is shown in Figure. 4 . The autocorrelation trace or the interferogram was measured under the following experimental conditions: The beam charge was $180 \mathrm{pC}$ and the electron beam energy was $13.4 \mathrm{MeV}$. The mixing angle initially was set to $\sim 5.3^{\circ}$. There were 40 micro-pulses in the pulse train at $1 \mathrm{MHz}$ with a repetition rate of $1 \mathrm{~Hz}$. The RTI interferogram can either be displayed in a digital interface or an analog output. We used the analog output, and the interferogram was displayed in an oscilloscope. The averaging was set to 4 samples (4 macropulses at $1 \mathrm{~Hz}$ ). The quadrupoles Q1 and Q2 at the entrance of the EEX beamline were set to $-0.699 \mathrm{~A}$ and $0.798 \mathrm{~A}$, respectively. This quadrupole setting was chosen based on measuring the CTR from X24 using a separate pyrodetector. At this quadrupole setting, the pyro-signal was maximized indicating (a) adequate CTR was available for the RTI measurements and (b) the bunch length of the electron beam was the typically sub-mm, which is within the frequency range of the RTI. After the RTI measurement, a Martin-Puplett interferometer was used to measure the autocorrelation of the CTR from X24.

The autocorrelation trace of the RTI along with that of the Martin-Puplett interferometer (MPI) is shown in Figure. 5. Although the RTI has only 32 channels, by remotely moving one of the mirrors (mirror d in Fig. 2), a complete autocorrelation can be determined. We moved the mirror in steps of $0.4 \mathrm{~mm}$ to obtain several traces and then combined them together to generate the trace shown in Fig. 5. In order to get an autocorrelation trace on the scope directly, an interferogram was taken at large path difference ( where no interference is expected) to measure the background and stored in memory. The actual autocorrelation signal was then divided by the background to obtain the interference pattern. The FFT of the autocorrelation data and a comparison with that of the Martin-Puplett data are shown in Fig. 6. The RTI has a low-frequency cut-off at $0.25 \mathrm{THz}$ and the MPI at around $0.1 \mathrm{THz}$.

Finally, the RTI was used to measure bunch lengths for different quadrupole settings using EEX, and the results were compared with streak-camera results, as shown in Fig. 7 . The bunch length is estimated as follows: first, the discrete autocorrelation signal is converted to a continuous trace by fitting a Gaussian curve through the center of each pixel element. 
The FWHM of the spatial autocorrelation trace is then calculated from the Gaussian fit.

The temporal FWHM of the signal is determined by a simple formula: $t_{F W H M}=\frac{N \sin (\theta) \delta x}{K c}$, where $\theta$ is the mixing angle, $\delta x=500 \mu \mathrm{m}, c$ is the speed of light, $\mathrm{N}$ is the FWHM of the autocorrelation trace and $\mathrm{K}$ is a factor depending on the shape of the pulse. For our analysis we assumed a Gaussian beam profile for which K is 1.414. Other experiments have shown this is a good assumption as long as the space-charge effect is negligible, coherent synchrotron radiation is low and there is no energy-time correlation on the incoming beam ${ }^{14}$.

The streak camera at the A0PI was operated in synchroscan mode in which all the micropulses are synchronously summed and included slew and jitter effects. The streak camera data were taken with the synchronous sum of 60 bunches, and averaged over 25 shots. The statistical uncertainty is a much smaller contribution than the estimated $10 \%$ uncertainty in the chromatic bandwidth correction term for the system.

\section{B. Discussion}

The autocorrelation measured from the RTI agrees well with the autocorrelation obtained from the MPI measurements, as seen in Fig. 5. The operation of the MPI and bunch length measurement using MPI are mentioned in detail elsewhere ${ }^{15}$. Since the RTI and the MPI use the CTR signal, if we compare the width of the central peak in the autocorrelation the agreement is good, but there is discrepancy in the zero-crossing of the autocorrelation wings, where the lower frequency information is contained. This is possibly because of the diffraction effects due to the difference in size of the detectors. The MPI uses $3 \mathrm{~mm}$ x 3 $\mathrm{mm}$ detectors while the RTI uses $1 \mathrm{~mm}$ x $0.5 \mathrm{~mm}$ detectors. This difference in size leads to a different low frequency response as seen in the frequency plot in Fig. 6. The observed attenuation due to diffraction at longer wavelength is consistent with the calculation ${ }^{16}$.

The RTI measurements were also compared to bunch length measurements from the streak camera. There is a good agreement between the RTI and the streak camera for bunch lengths (FWHM) less than 1.0 ps as shown in Fig. 7. While the RTI uses far infrared CTR, the streak camera uses the incoherent optical transition radiation. At longer bunch lengths, the discrepancy between the two techniques becomes large due to the poor low frequency response of the RTI detector. Preliminary analytical modeling shows that assuming a flat response for the pyrodetectors and low frequency cutoff at $0.25 \mathrm{THz}$, the RTI 
could underestimate the bunch length by $25 \%$ at $~ 1$ ps FWHM. However, for even shorter bunch lengths this effect would be reduced. Moreover, the simple estimation of the bunch length from the autocorrelation central peak excludes the form factor or the shape of the bunch, which could play a significant role. Further investigations are planned to study this effect. Similar behavior has been observed between the Martin-Puplett interferometer and the streak camera. Also at shorter bunch length the streak camera reaches its resolution limit leading to a larger uncertainty in the bunch length measurement.

\section{Resolution limit of the RTI}

The resolution limit of the RTI depends on the transverse beam size and the mixing angle. The transverse beam size at the input of the RTI has to be about $2 \mathrm{~cm}$ to cover the entire detector length $(16 \mathrm{~mm})$. This could be a limitation for high-energy beam as the CTR cone is restricted to an angle $\sim \frac{1}{\gamma}$, where $\gamma$ is the relativistic factor. However, the CTR spot size can always be increased by using a telescope, albeit with some loss due to absorption and transport at $\mathrm{THz}$ frequencies. Also, the mixing angle in the RTI has to be decreased for shorter bunches. Ultimately, there is a tradeoff between the resolution in time versus the autocorrelation width. From a practical point of view, the angle has to be such that it accommodates the 4- $\sigma$ width of the autocorrelation trace. For the A0 photoinjector the lower limit on the mixing angle would be $1.2^{\circ}$. The uncertainty in the measurement for the experimental setup at $7.2^{\circ}$ is around \pm 100 fs. An accurate bunch-length measurement could be achieved by using a shutter to close one arm while opening the other and vice versa. This would deduct effects from the lateral transverse misalignments of the two lobes on the detector, which could improve the accuracy of the bunch length measurements. Also, fitting the autocorrelation trace in time is more advantageous than using the FFT reconstruction since it is difficult to collect all of the frequency information.

As in a typical temporal autocorrelation, the RTI has a number of sources of error for bunch length determination. The loss of long wavelengths due to diffraction at the source is a problem as well as the loss of short wavelengths due to finite transverse beam size, or finite apertures. But these errors could be corrected using a mathematical function based 
on previous electron beam measurements. Additional spectral corrections originate from the THz transport optics, RTI component response, and absorption efficiency of the detector and associated windows. These factors are associated with the finite sensitivity and dynamic range of the detector. Therefore, careful treatment of each component through analysis, calibration, simulation and further experimentation would allow for meaningful, informative data reconstruction in a real-time environment.

Finally, though the RTI could operate in a single-shot mode, the charge of the micropulse must be in excess of $1.2 \mathrm{nC}$ to get an appreciable signal or the bunch length has to be much shorter than $200 \mathrm{fs}$, which is hard to obtain at the A0 photoinjector. The number of bunches at $\mathrm{A} 0$ can be varied from 1 to 60 micropulses per macropulse. At low charge $(\sim 250 \mathrm{pC})$ , the RTI needed around 40 bunches for a good measurement while at higher charges $(\sim 1$ $\mathrm{nC})$ it needed around 5-6 bunches per macropulse for appreciable signal. This is consistent with the coherent nature of the CTR which scales as $\sim \frac{Q^{2}}{\sigma_{z}}$, where $Q$ is the charge in the pulse and $\sigma_{z}$ is the bunch length of the pulse. A factor of 4 increase in charge increases the signal level by a factor of 16 - roughly equivalent to the reduction in the number of pulses needed to record a trace. Although this could be a limitation for using the RTI in accelerators where the number of micropulses per bunch is very low, a redesign using more sensitive detectors would overcome this limitation. For modern accelerators and FELs based on superconducting accelerator technology, we do not expect this to be a limitation for the RTI.

\section{CONCLUSION}

The real-time interferometer (RTI) has been commissioned at the A0 photoinjector. For the first time, the autocorrelation was promptly recorded in real-time as compared to several minutes for the Martin-Puplett interferometer. It has sensitivity to the variation in bunch length of the electron beam in the sub-mm regime. Ideally, the RTI does not have any movable parts and even the geometry of the setup constrains any misalignment in the mixing angles. It is relatively compact, easily portable, and allows the machine operator to tune the accelerator by rapid estimation of the autocorrelation width thus acting as a real-time bunch-length monitor .

The RTI could be improved in the following ways: (a) larger optics to avoid diffraction 
(b) increase the number of channels in the pyro-detector array allowing one to image the complete autocorrelation trace in a single shot and (c) remote control of the mirror to control the mixing angle and thus adjust the resolution of the instrument on-line. These additions will be implemented in the next design of the RTI. The applicability of the RTI could be explored at high energies under different beam parameters at the Free-Electron Laser in Hamburg (FLASH) facility at DESY, the Next Linear Collider Test Accelerator (NLCTA) and the Facility for Advanced Accelerator Experiment Tests (FACET) facility at Stanford Linear Accelerator Center, the Advanced Superconducting Test Accelerator facility (ASTA) at Fermilab, and the Accelerator Test Facility (ATF) at Broohaven National Lab.

\section{ACKNOWLEDGMENTS}

The authors are indebted to the A0 technical support team and the Radiabeam technical support. We acknowledge Gentec-EO for their help with the electronics. We thank M. Church, H. Edwards, M. Wendt, V. Shiltsev, and P. Piot for their interest and encouragement. We thank E. Hemsing for his calculations. We also thank J. Moody, P. Musumeci, J. Rosenzweig, R. Tikhoplav, G. Travish, and U. Happek. The work was supported by the Fermi Research Alliance, LLC under the U.S. Department of Energy and by US DOE SBIR Grant DE-FG02-07ER84814.

\section{REFERENCES}

${ }^{1}$ P. Emma, R. Akre, J. Arthur, R. Bionta, C. Bostedt, J. Bozek, A. Brachmann, P. Bucksbaum, R. Coffee, F. Decker, Y. Ding, D. Dowell, S. Edstrom, A. Fisher, J. Frisch, S. Gilevich, J. Hastings, G. Hays, P. Hering, Z. Huang, R. Iverson, H. Loos, M. Messerchmidt, A. Miahnahri, S. Moeller, H. Nuhn, G. Pile, D. Ratner, J. Rzepiela, D. Schultz, T. Smith, P. Stefan, H. Tompkins, J. Turner, J. Welch, J. Wu, G. Yocky, and J. Galayda, Nature photonics, vol. 4, no. 9, pp. 641-647, 2010.

${ }^{2}$ I. Wilke, A. MacLeod, W. Gillespie, G. Berden, G. Knippels, and A. Van Der Meer, Physical review letters, vol. 88, no. 12, p. 124801, 2002.

${ }^{3}$ M. Hüning, A. Bolzmann, H. Schlarb, J. Frisch, D. McCormick, M. Ross, T. Smith, and 
J. Rossbach, Proceedings of the 27th International Free Electron Laser Conference (FEL 2005), p. 538, 2005.

${ }^{4}$ Z. Huang, K. Bane, Y. Ding, and P. Emma, Physical Review Special Topics-Accelerators and Beams, vol. 13, no. 9, p. 092801, 2010.

${ }^{5}$ G. Andonian, A. Murokh, M. Ruelas, A. Ovodenko, R. Tikhoplav, S. Reiche, and D. Dooley, International Particle Accelerator Conference 2010, 2010.

${ }^{6}$ L. Frohlich and O. Grimm, TESLA FEL Report, vol. 2, 2005.

${ }^{7}$ J. Ruan, A. Johnson, A. Lumpkin, R. Thurman-Keup, H. Edwards, R. Fliller, T. Koeth, and Y. Sun, Physical Review Letters, vol. 106, no. 24, p. 244801, 2011.

${ }^{8}$ U. Happek, A. J. Sievers, and E. B. Blum, Phys. Rev. Lett., vol. 67, pp. 2962-2965, Nov 1991.

${ }^{9}$ D. Sütterlin, D. Erni, V. Schlott, H. Sigg, H. Jäckel, and A. Murk, Review of Scientific Instruments, vol. 81, no. 10, p. 104702, 2010.

${ }^{10}$ N. I. Agladze, J. M. Klopf, G. P. Williams, and A. J. Sievers, Appl. Opt., vol. 49, pp. 32393244, Jun 2010.

${ }^{11}$ S. Wesch, B. Schmidt, C. Behrens, H. Delsim-Hashemi, and P. Schmser, Nuclear Instruments and Methods in Physics Research Section A: Accelerators, Spectrometers, Detectors and Associated Equipment, vol. 665, no. 0, pp. 40 - 47, 2011.

${ }^{12}$ R. Lai, U. Happek, and A. Sievers, Physical Review E, vol. 50, no. 6, pp. 4294-4297, 1994.

${ }^{13}$ A. Lumpkin, A. Johnson, J. Ruan, J. Santucci, Y. Sun, R. Thurman-Keup, and H. Edwards, Physical Review Special Topics-Accelerators and Beams, vol. 14, no. 6, p. 060704, 2011.

${ }^{14}$ J. Thangaraj, R. Thurman-Keup, J. Ruan, P. Piot, A. Johnson, H. Edwards, J. Santucci, A. Lumpkin, Y. Sun, M. Church, and T. Maxwell, Proceedings of the 27th International Free Electron Laser Conference (FEL 2005), p. MOPC12, 2011.

${ }^{15}$ R. Thurman-Keup, R. Fliller, and G. Kazakevich, Proceedings of Beam Instrumentation Worskhop 2008, 2008.

${ }^{16}$ A. Murokh, J. Rosenzweig, M. Hogan, H. Suk, G. Travish, and U. Happek, Nuclear Instruments and Methods in Physics Research Section A: Accelerators, Spectrometers, Detectors and Associated Equipment, vol. 410, no. 3, pp. 452 - 460, 1998. 

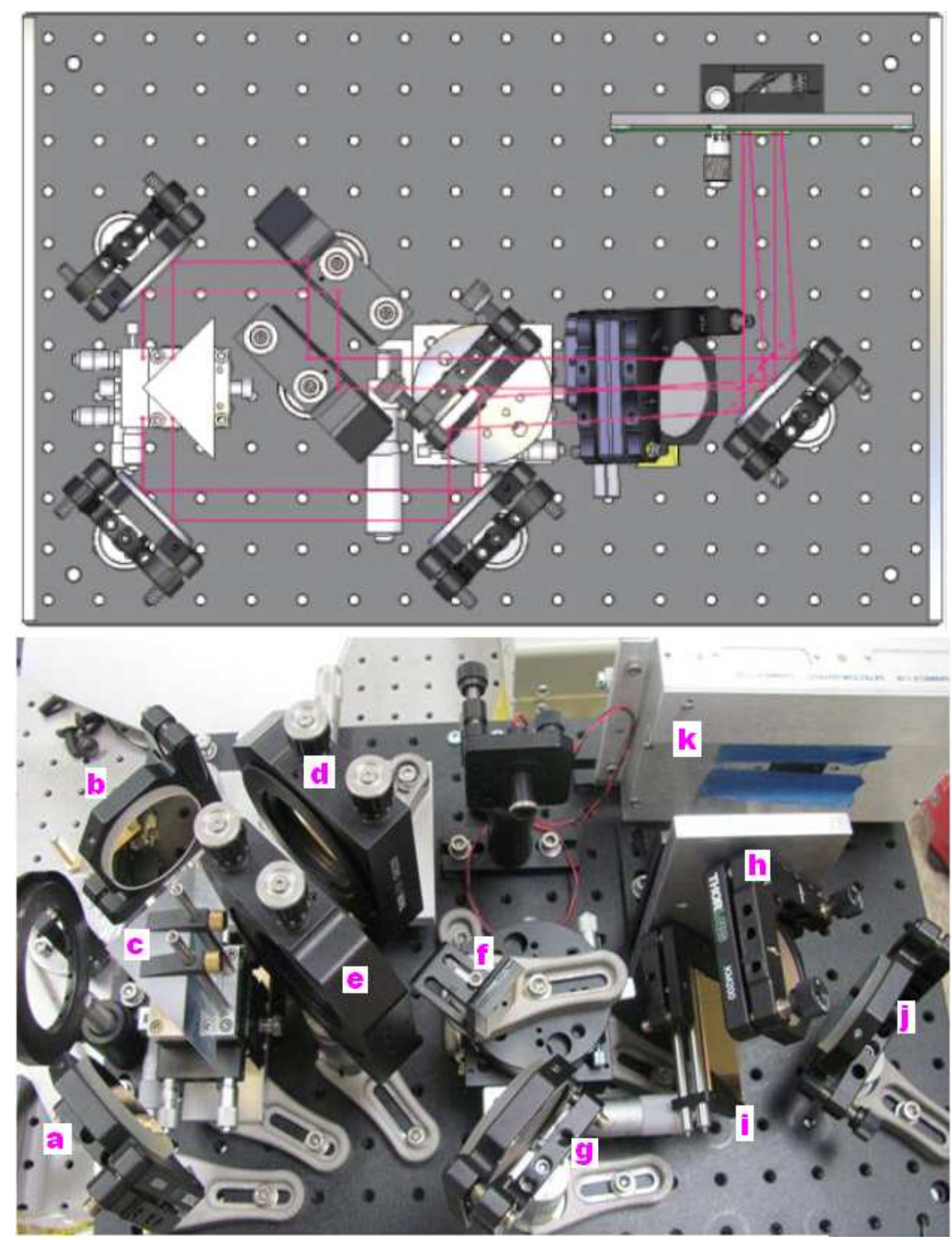

FIG. 2. The top figure shows the engineering design of the RTI with the optics. The lines trace the rays of the radiation through the optics. The bottom figure shows the actual experimental setup of the RTI. The input beam is split by a wedge splitter (c) and then sent to mirrors (a,b). One of the split signal travels to the movable stage (d) and then is reflected back through the mirrors $(\mathrm{e}, \mathrm{h})$ to the cylindrical mirror (i). The other signal from mirror (a) travels to mirror (g) and is reflected by a angle-adjustable mirror (f), which is sent to the flat mirror (h). After both signals arrive at the cylindrical mirror (i), they are focussed with the cylindrical mirror (i) and directed to the pyro-detector array with the flat mirror $(\mathrm{j})$. The signal from the pyro-detector array $(\mathrm{k})$ is sent to an oscilloscope. 


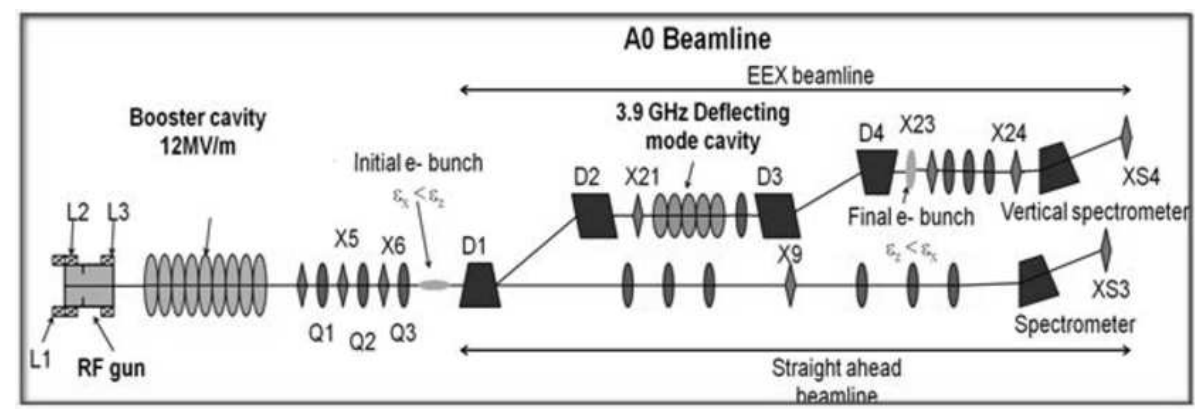

FIG. 3. Experimental setup of the A0 photoinjector facility. The electrons generated by the RF gun are accelerated by the booster cavity to $14 \mathrm{MeV}$. The beam is then focused using the quadrupoles (Q1,Q2,Q3) before it is sent through the EEX beam line. After going through the dogleg section of the beamline, the beam hits a metal screen at X24 and generates Coherent Transition Radiation (CTR) which is transported to the RTI. The quadrupoles are marked as ovals and the diagnostic stations are marked as diamonds. D1, D2, D3 and D4 are dipole magnets used to bend the beam. 


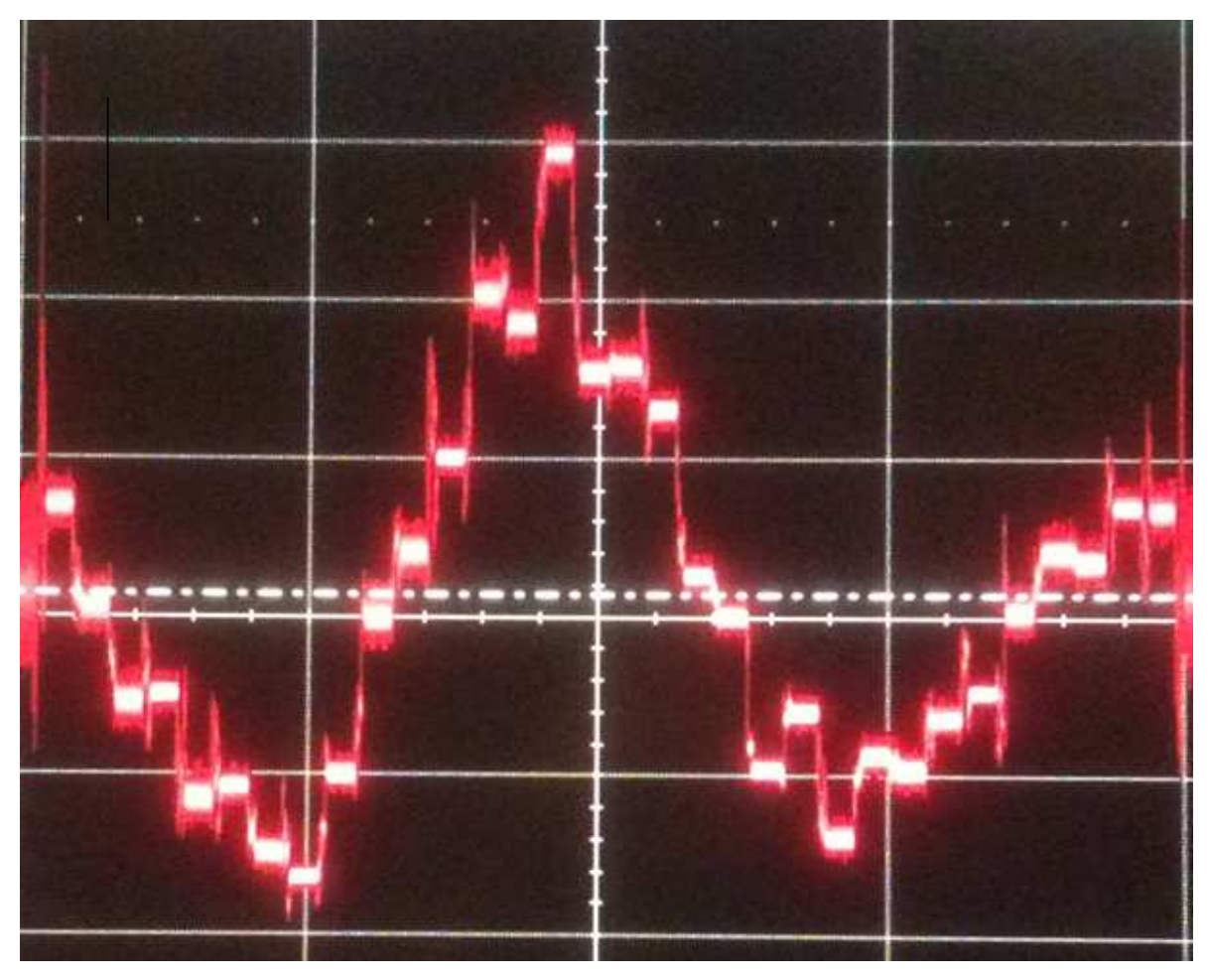

FIG. 4. The raw oscilloscope trace obtained from the 32-channel detector array of the RTI showing the central part of a typical autocorrelation. The x-axis is time $(1 \mathrm{~ms} / \mathrm{div})$ and the $\mathrm{y}$-axis is volts $(200 \mathrm{mV} / \mathrm{div})$. Each element in the array is illuminated depending on the strength of the autocorrelation signal at that element. By counting the number of pixels in the central(FWHM) width, for a given mixing angle, the bunch length can be estimated. The trace was obtained from the Coherent Transition Radiation, when a $13.4 \mathrm{MeV}$ electron beam hits a foil. The number of pulses in the bunch train was 40 bunches with each pulse carrying $180 \mathrm{pC}$. 


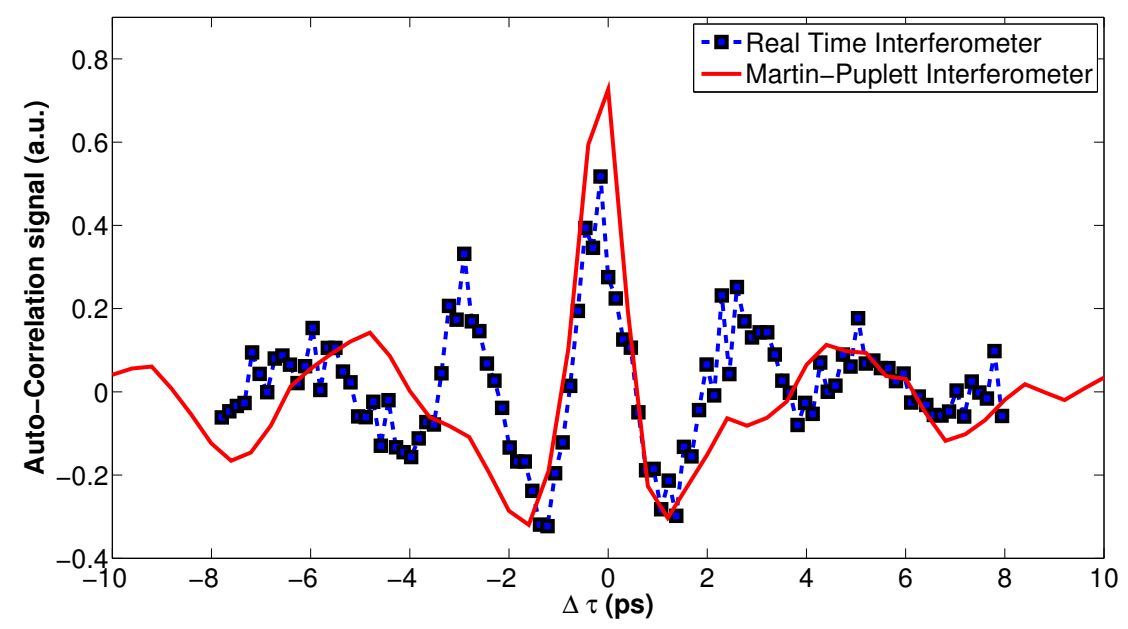

FIG. 5. Autocorrelation trace obtained from the Real Time Interferometer (RTI) compared with the autocorrelation trace obtained from the Martin-Pupplett interferometer (MPI). The agreement in the central part of the autocorrelation - which determines the bunch length - is good while the sides of the autocorrelation differs due to the low-frequency response of the detectors in MPI and the RTI.

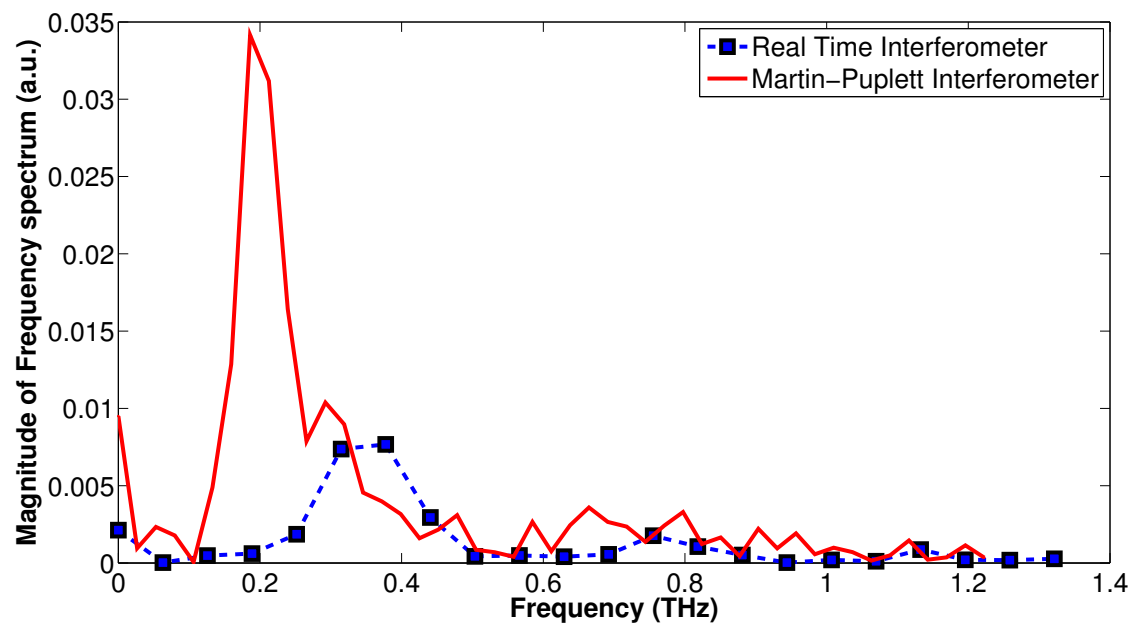

FIG. 6. Fast Fourier Transform of the autocorrelation trace obtained from the RTI and MPI. While the high frequency cut-off between the MPI and the RTI agrees well, the low frequency response is different. The difference in the size of the detector might explain the low frequency response. 


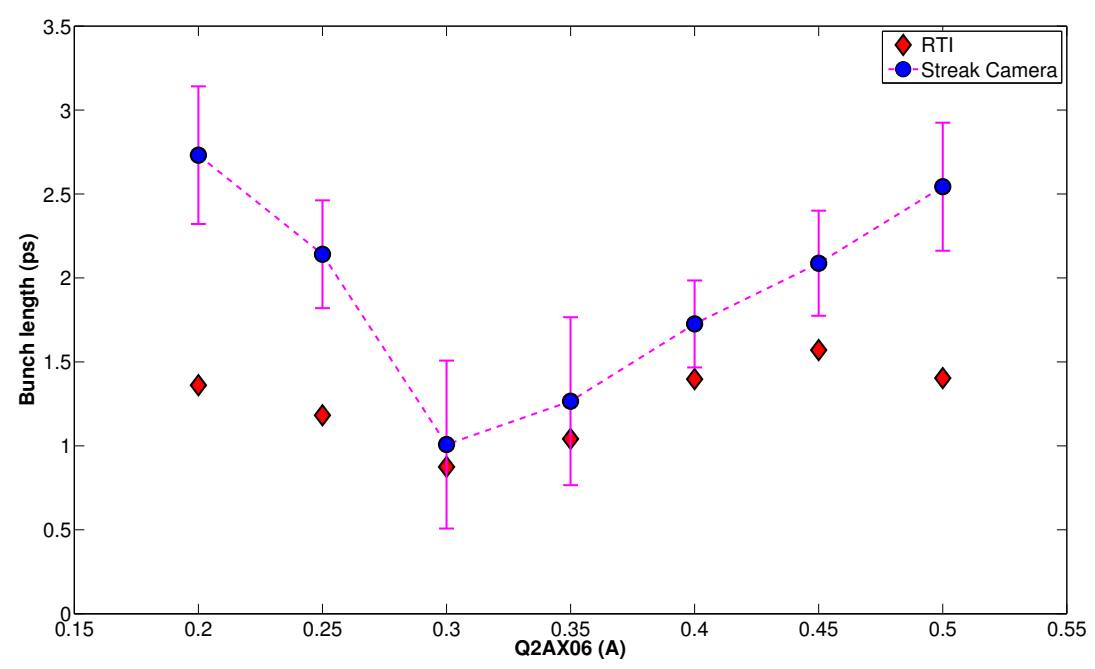

FIG. 7. A comparison of the bunch length (FWHM) obtained from the streak camera and the RTI for various quadrupole settings of Q2AX06 (labeled Q3 in Fig. 3). At longer bunch lengths, the discrepancy between the RTI and the streak camera is due to the poor low-frequency response of the RTI. At the shorter bunch lengths, the streak camera approaches its resolution limit and hence a larger uncertainty. Moreover, the simple estimation of the bunch length from the RTI autocorrelation trace excludes the form factor or the shape of the bunch, which could play a significant role as well. 